\title{
Measures of uncertainty in power split systems
}

\author{
Serhan Ozdemir * \\ Artificial Iytelligence and Design Lab, Mechanical Engineering Department, Izmir Institute of Technology, 35437 Izmir, Turkey
}

Received 27 August 2004; received in revised form 24 March 2006; accepted 4 April 2006

Available online 5 June 2006

\begin{abstract}
This paper discusses the overlooked uncertainty inherent in every transmission. The uncertainty aspect has been often, for the sake of clarity, ignored. Instead, mechanical transmissions have been characterized traditionally by their transmission efficacies. It is known that transmission localities are sources of power loss, depending on many factors, hence sources of uncertainty. Thus each transmission of power should not only be designated by a constant of efficiency but also by an expression of uncertainty, reflecting the probability of transmission. Furthermore, Shannon's and Renyi's expressions of entropy have been used to quantify this so-called transmission uncertainty. The entropy of a transmitting unit is given in these two forms and then compared. Practical formulations for flow optimization are given.
\end{abstract}

(c) 2006 Elsevier Ltd. All rights reserved.

Keywords: Power transmission; Entropy; Shannon's entropy; Renyi’s entropy; Uncertainty

\section{Introduction}

Traditionally, mechanical transmissions have been characterized by their transmission efficacies. This efficacy is given by the ratio of the output to the input of the transmitted entity, generally either power or torque. Unfortunately, the power transmission phenomenon is slightly more complex than that. The simplification of the calculation of the power transfer has both up and downsides to it. This process of defining the power "junction" by a constant of efficiency simplifies the computations and gives the designer an upper hand by allowing him to focus on more complicated matters. The downside to this simplification is that power transmission is a complicated matter itself.

Each transmission of power or torque should be regarded in its own right. These junctions could safely be thought of as portals where an entity of two different magnitudes coexist. As any designer would agree, each of these portals is a source of uncertainty. Thus each transmission of power, or torque for that matter, should not only be designated by an efficiency but also by an expression of uncertainty. This statement of uncertainty would reflect the designers trust in the transmission.

\footnotetext{
* Tel.: +90 232750 6585; fax: +90 2327506505 .

E-mail address: serhanozdemir@iyte.edu.tr
} 
To clear the issue further, one must understand that a constant of efficiency is nothing but an average value of previous experiments or a catalogue value of the manufacturer. This is to say that under all conditions, a certain percentage of power will always be transferred. But this is an oversimplification. Alongside this constant, a term of uncertainty also ought to be given. Considering the fact that power transmission is an interplay of many factors, from temperature to lubrication etc., transmission phenomenon is converted into a stochastic event. This stochasticity is expressed in entropy, which has deep roots in science, and can be expressed by information theory.

Entropy has been in use for decades in a wide range of areas. It has its origins back in the 19th century, dating back to Boltzmann. His formulation of entropy was meant to quantify the disorder that every physical system strives to maximize. Considered as the cornerstone in statistical mechanics, it has been put to use from black holes to chemistry. In time, various new formats have also been introduced. Shannon and Renyi are just to name a few. Another one is gaining recognition and known as Tsallis entropy [1]. Tsallis is different from the conventional entropy definition in that it is non-extensive, meaning the entropy of an entire system no longer equals the sum of the entropies of its various parts. This property is displayed by the systems on the verge of chaos. Tsallis entropy has already been applied to research from the locomotion of microorganisms to the collisions of subatomic particles.

To be specific, examples can be enumerated on the general uses of entropy. Absolute maximum entropy principle, for example, is invoked in [2] to construct an automatic modeler with the least loss of information, where transition is from continuous to discrete random variables. Another modeling is performed in [3] in order to optimize mixing and particle sizes. One aspect to mention here is that transition function entropy is in continuous format. Even the web navigation, informally known as surfing, has been modeled by entropy [4]. Each navigation leaves a trail which is considered a stochastic variable since, it is claimed, users get disoriented in terms of their original query. Then entropy is used, alongside a Markovian approach, to characterize the user navigation.

In the following sections, reasons leading to entropy in a power transmission are stated. Then basic definitions of entropy and a comparison of formats are given. In later sections, formulations of optimization and interpretations are also provided.

\section{Sources of uncertainty}

Practically every connection in a mechanical system is a source of entropy. The ones with the highest entropy are

- Belt drives

Angular uncertainty due to slippage.

- Clutch mechanisms

Similar to belt drives.

- Chain drives

Variation in linear chain speed, known as chordal speed variation.

- Gear mesh

Minor uncertainty due to power loss and possible backlash, favored.

For each and every one of these drives, various entropies could be defined. For example, belt drives and clutches could be designated by a positional entropy, whereas a chain drive by a speed entropy etc. The following is a generic, drive-wise non-specific formulation.

\section{Entropy as a measure of uncertainty}

Shannon in his seminal work in 1948 has described a formula of uncertainty. He devised a way to account for the uncertainty of any received message, say, in bits. The idea was to quantify the possibility of error due to channel noise. Information theory is naturally beyond the scope of this work. 
A summary of the properties of entropy are as follows [5]:

- It is the unit of uncertainty.

- Outcomes with zero probability do not change the uncertainty. By convention, $0 \log 0=0$.

- When the outcome of an event is certain, the entropy is zero.

- When all the events are equiprobable, the entropy is maximal.

- Entropy increases as the number of events increase.

- The entropy does not depend on the sequence of events.

- The entropy is the only function appropriate for the uncertainty measure (the uniqueness theorem).

A brief formulation of entropy is expressed below, Knessl [6]. The uncertainty by Shannon entropy was given by

$$
H=-\sum_{i=1}^{M} P_{i} \log _{2} P_{i}
$$

where $M$ : number of symbols, $P_{i}$ : probability of a symbol appearing, $H$ : uncertainty.

Here, the base 2 logarithm refers to the two states of operation. The number of symbols are also limited to 2. These two symbols represent signal and no signal states. In this paper, they will mean "power pass" and "no pass" states. From now on, when a transmission is given by a constant of efficiency, this constant will be considered as the probability of power flow. Suppose that a unit has a probability of $\eta$ (efficiency) to transmit power, that is just another way of saying that the power will be absorbed with a probability of $(1-\eta)$.

\subsection{Entropy in power transmission}

Characterizing a discontinuity for the power to flow in entropy terms will have the following characteristics:

1. Considers transmission as a probabilistic event.

2. Penalizes transmissions at low efficiency points more than basic algebra.

3. Gives an analytical expression to optimize for the power to flow.

Transmission of power is indeed a probabilistic event. Since temperature, material characteristics etc., at contact surfaces all vary, so does the transmission efficiency. This makes the constant of efficiency a stochastic entity, and the transferred power a probabilistic phenomenon. Logarithmic formulation of uncertainty penalizes low efficiencies further than simple algebric operations would do. The last part is especially important when the power has more than one route to flow. This is true of hybrid transmissions, where motion is split and partly transmitted through, say, over a planetary gear set and partly through a pulley-belt mechanism. Such a performance measure would fit well its niche in an application such as Ozdemir and Schueller [7]. The percentage to follow through the gear set will be determined by an entropy minimization process.

For a power line with power transmission localities, it would be proper to define a general "Total Transmission Entropy" or TTE

$$
\mathrm{TTE}=-\sum_{k=1}^{B} \sum_{j=1}^{N} \sum_{i=1}^{2} P_{i} \log _{2} P_{i}
$$

where $N$ : total number of elements that can be assigned an efficiency of transmission on a single branch, $B$ : total number of branches that power is transmitted through, $P_{i}$ : transmitted power through a designated route.

It should be noted that, throughout this paper, the input power is always taken unity. So $P_{i}$ along any branch will be less than unity.

In multipath torque transmission, the logical idea would be running most of the power along the most efficient line. The upper limiting factors could probably be strength, and/or design considerations. Obviously, for two routes of varying efficiencies, power should be split proportionally to the efficiencies. 
TTE could be simplified with no loss of generality:

$$
\mathrm{TTE}=-\sum_{k=1}^{B} \sum_{i=1}^{N} P_{i} \log _{2} P_{i}
$$

where only "pass" state is considered, and every branch is given by the sum of the individual transmission entropies.

\section{Optimal power distribution with the least uncertainty}

By integrating the uncertainty term in a cost function, a real time route optimization could be scheduled. Consider for example the cost function

$\operatorname{Min} J=F(p)-\sum_{i=1}^{B} \eta_{i} w_{i} P_{\text {in }} \log _{2}\left(\eta_{i} w_{i} P_{\text {in }}\right)$

Subject to

(i) $F(w)$

(ii) $\sum_{i=1}^{B} w_{i}=1$

where $F(p)$ could be the cost of running power through a certain path in, say, fuel, or amount of vibration etc., and $F(w)$ is scheduled power split for a power split transmission, and $w_{i}$ is the percentage of input power through $i$ th path.

Such a cost function would take into consideration the uncertainty as well as all the design requirements. By weighting these separate factors, by the order of importance or cost, an optimal power split policy may be obtained.

\subsection{Lower threshold of power split}

The output power for a $B$ branch system may be described by

$$
P_{\text {out }}=-P_{\text {in }} \sum_{i=1}^{B} \eta_{i} w_{i}
$$

Now let us consider again Fig. 1 [7]. Power branching out in two and, to simplify the matters, each branch contains just a single unit, the output power could be written as

$$
P_{\text {out }}=P_{\text {in }}\left(w_{1} \eta_{1}+w_{2} \eta_{2}\right)
$$

The TTE for this case is

$$
\mathrm{TTE}=-\left(w_{1} \eta_{1}\right) \log _{2}\left(w_{1} \eta_{1}\right)-\left[\left(1-w_{1}\right) \eta_{2} \log _{2}\left(\left(1-w_{1}\right) \eta_{2}\right)\right]
$$

Worst case power split ratio could now be found by deriving TTE with respect to $w\left(w_{1}\right)$ and then setting this to zero. This value of $w_{1}$ will give the lower threshold of power split through the 1st path.

$$
\frac{\mathrm{d}(\mathrm{TTE})}{\mathrm{d} w_{1}}=0
$$

Hence,

$$
\eta_{2} \log _{2}\left[\left(1-w_{1}\right) \eta_{2}\right]-\eta_{1} \log _{2}\left(w_{1} \eta_{1}\right)+\eta_{2}-\eta_{1}=0
$$

Eq. (9) could be restated in a rational form as

$$
\exp \left(\eta_{1}-\eta_{2}\right)=\frac{\left[\left(1-w_{1}\right) \eta_{2}\right]^{\eta_{2}}}{\left(w_{1} \eta_{1}\right)^{\eta_{1}}}
$$




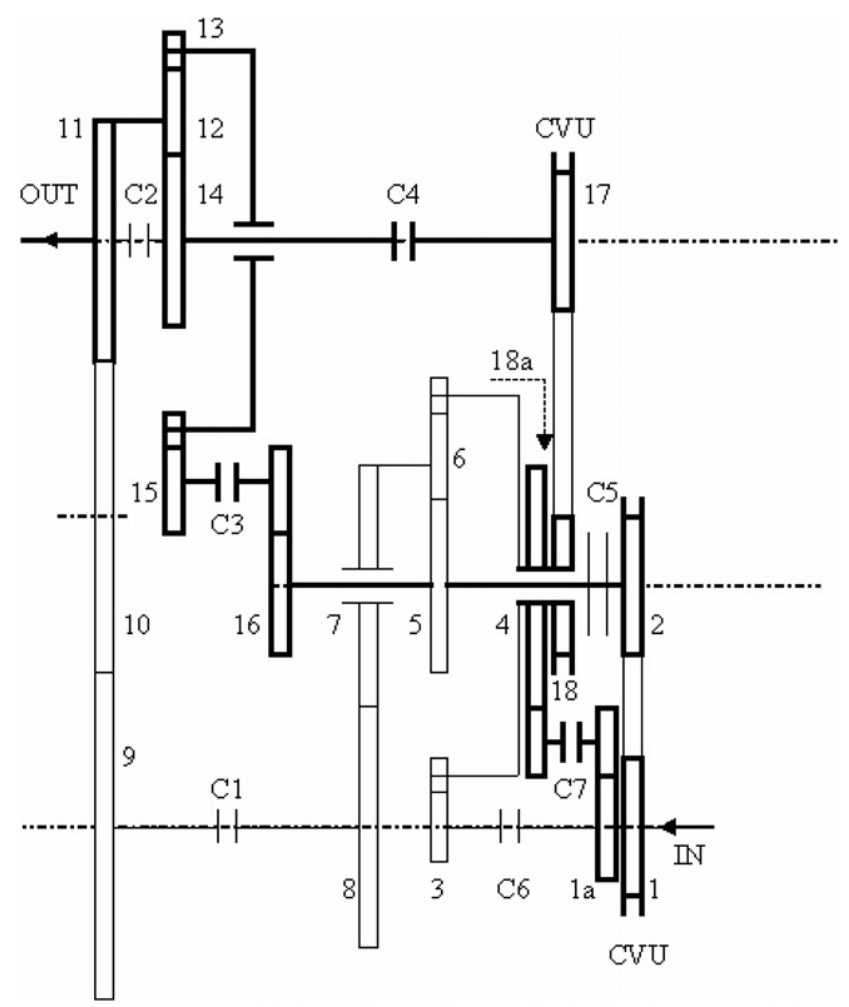

Fig. 1. A two-branch power split scenario, where $\mathrm{C} 1, \ldots, \mathrm{C} 7$ : clutches; CVU: continuously variable units; 1,2,17, 18: variable pulleys; $3,4, \ldots, 15,16$ : gears, [7].

Optimal power split ratio should remain well away from the worst case scenario found by the above formula

$$
w_{\mathrm{opt}} \gg w_{1}
$$

As an example case, suppose now that for the same two-branch system, first path has a 0.95 and the second path has 0.45 efficiency. Fig. 2 shows that under these circumstances, the worst case appears around when $35 \%$

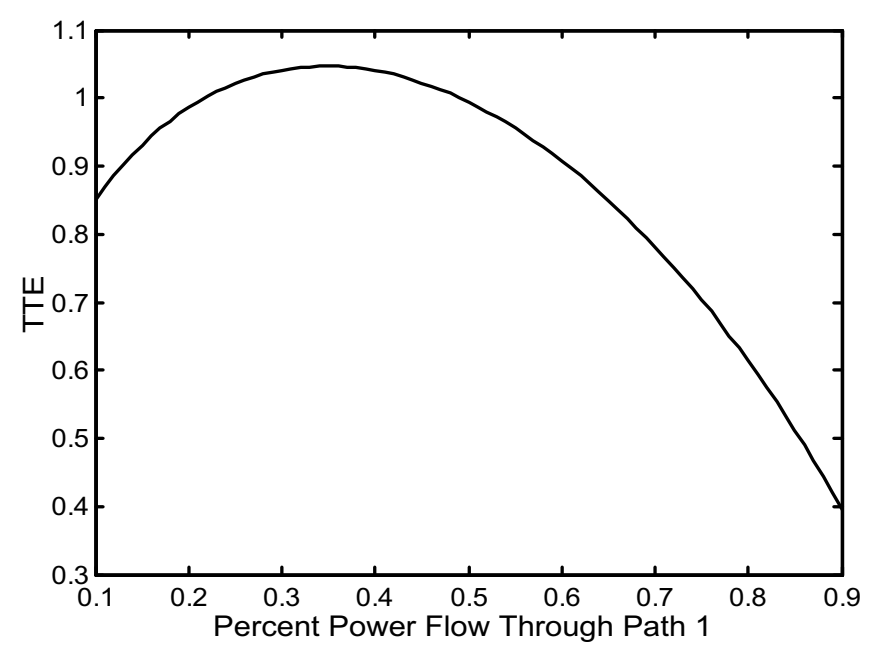

Fig. 2. Total transmission entropy vs. $w_{1}$. 
of power flows through first route. This rate also represents the highest uncertainty. As more and more power is transmitted through line 1, uncertainty approaches zero.

Now, another entropy format, Renyi entropy is considered. Renyi is a lesser known expression of entropy. It can be used to assess the uncertainty of incomplete system of events, and is given below for comparison:

$$
H=\frac{1}{1-k} \log \left[\sum p^{k}\right]
$$

where $k$ is the Renyi entropy parameter and $p$ is probability as before. Renyi and Shannon expressions are, in fact, related. In the limit, as $k$ tends to 1, Renyi's entropy approaches Shannon formulation.

Now re-writing TTE in Renyi format for the same two-branch example

$$
\begin{aligned}
& \mathrm{TTE}=-\frac{1}{1-k} \log \left[\left(w_{1} \eta_{1}\right)^{k}+\left(\left(1-w_{1}\right) \eta_{2}\right)^{k}\right] \\
& \frac{\mathrm{d}(\mathrm{TTE})}{\mathrm{d} w_{1}}=0
\end{aligned}
$$

which simplifies into

$$
\eta_{1}\left(w_{1} \eta_{1}\right)^{k-1}-\eta_{2}\left(\left(1-w_{1}\right) \eta_{2}\right)^{k-1}=0
$$

Nevertheless, Eq. (14) does not produce any lower limits on the power flow ratio. As it can be seen in Fig. 3, entropy is gradually decreases as $w_{1}$ increases. This could partially be explained by the lack of convexity of the Renyi form. Renyi is straightforward in that there is no worst point of operation, a point of convexity. But rather, it imposes a penalty on every state of operation away from the trivial case, which is the flow through the most efficient path. Yet the acuteness of this penalty, as can be seen from Fig. 3, relents towards the increasing values of $w$. This contrasts with that of Shannon, in Fig. 2, in that slope gets steeper towards same values of $w$. In other words, the rate at which Shannon entropy penalizes on increasing split ratios is less than the rate by Renyi's.

\subsection{Consideration of individual entropies}

An example might be given to demonstrate how successive units on the same branch increase uncertainty compared to a single one. Curiously, this could be taken as an alternative definition of reliability. Consider, for

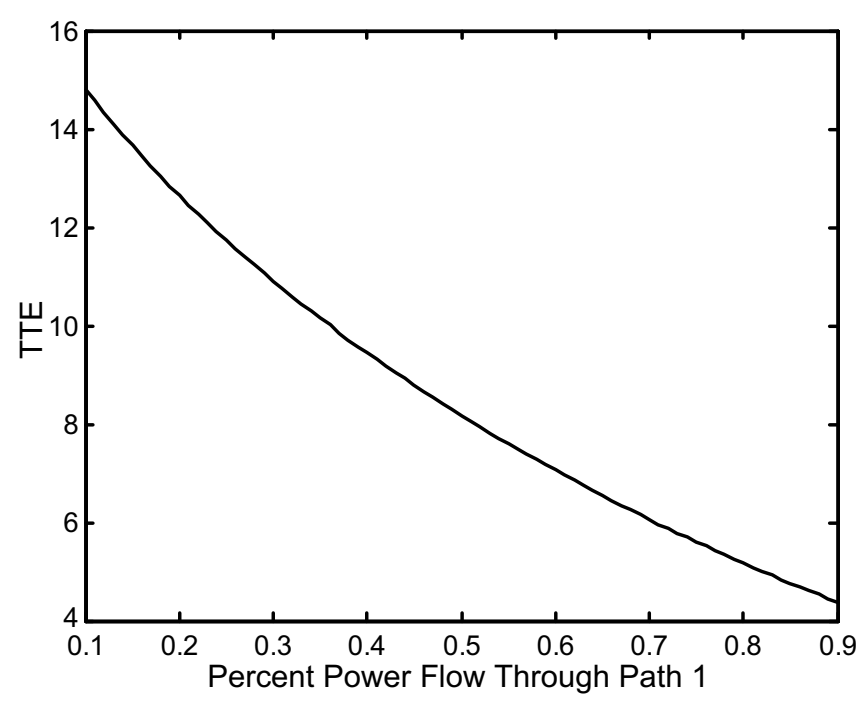

Fig. 3. Total transmission entropy of Renyi vs. $w_{1}$. 


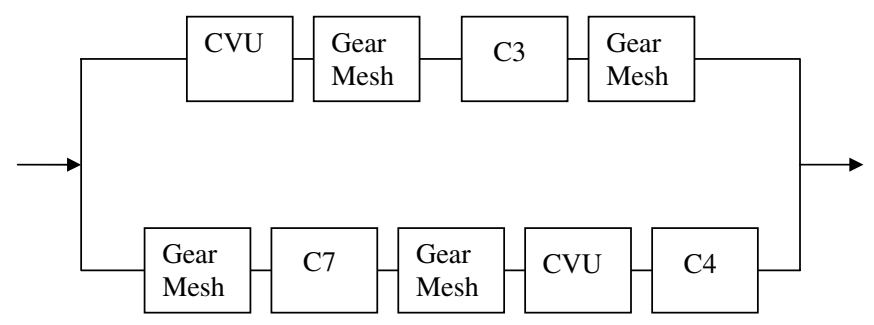

Fig. 4. Power flow diagram of Fig. 1.

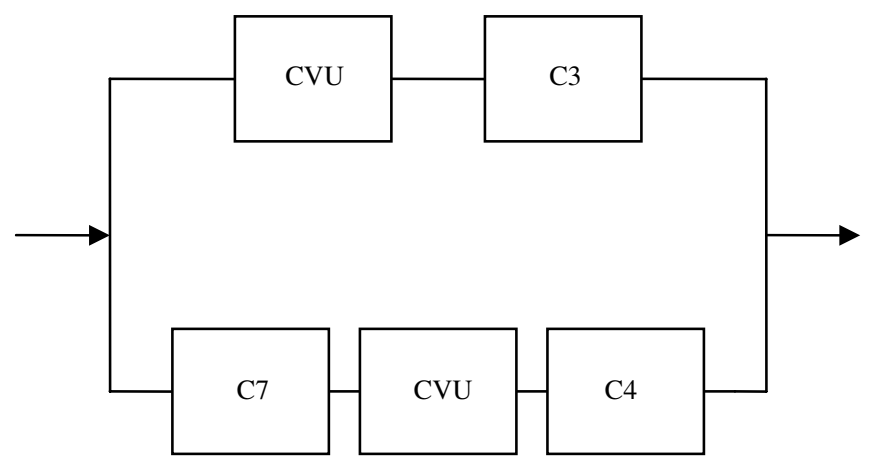

Fig. 5. The simplified power flow diagram of Fig. 4, with gear meshes omitted.

instance, Fig. 1, where a complicated two-branch power split mechanism is shown. An equivalent power flow diagram is given in Fig. 4. If gear mesh is assumed to be very efficient compared to the rest of the mechanisms, i.e. continuously variable units (cvu) and clutches, a simplified version could be used as in Fig. 5.

In Fig. 5, take for instance, the efficiencies of the cvu's and clutches as $\eta_{\mathrm{cvu}}=.90$, and $\eta_{\mathrm{clutch}}=.95$, respectively. By the definition in Eq. (3), Tables 1 and 2 may be constructed. Note that simply because there are more transmission localities in the lower path, uncertainty is augmented. The uncertainty in terms of the entropy is computed as follows:

Individual entropies, the sum, and the entropy of the unit by the resultant efficiency are given in Table 1. Upper path possesses the lowest uncertainty as expected due not only to the higher efficiency but also to less number of power transmittals. The calculations in Table 2 juxtapose a contrast that a slight difference in $\eta$ creates. This proves that logarithmic cost function indeed penalizes more than mere comparison. Both in traditional and entropy-based methods, power is split in proportion to either in efficiency, as in the first two columns in Table 2, or in entropy as in the remaining columns of the same table.

Table 1

Individual entropies and resultant efficiencies at various localities

\begin{tabular}{llllll}
\hline$E\left(\eta_{\mathrm{cvu}}\right)$ & $E\left(\eta_{\text {clutch }}\right)$ & $\begin{array}{l}\text { Upper branch } \\
\eta_{\text {res }}=\eta_{\text {cvu }} \eta_{\text {clutch }}\end{array}$ & $\begin{array}{l}\text { Lower branch } \\
\eta_{\text {res }}=\eta_{\text {cvu }} \eta_{\text {clutch }}^{2}\end{array}$ & $\begin{array}{l}\text { Upper branch } \\
\sum_{i=1}^{2} \eta_{i} \log \eta_{i}\end{array}$ & $\begin{array}{l}\text { Lower branch } \\
\sum_{i=1}^{3} \eta_{i} \log \eta_{i}\end{array}$ \\
\hline 0.1368 & 0.0703 & 0.855 & 0.812 & 0.2071 & 0.2774 \\
\hline
\end{tabular}

Table 2

Traditional efficiency-based and entropy-based recommended power split

\begin{tabular}{lllll}
\hline Upper branch & Lower branch & TTE & Upper branch & Lower branch \\
\hline $51 \%$ & $49 \%$ & 0.4845 & $57 \%$ & $43 \%$ \\
\hline
\end{tabular}


Results could be interpreted as follows:

- TTE regards serial transmission junctions with reduced trust.

- It suggests slightly more power transfer from serial branches to single unit (less uncertain) ones.

- It discerns among types of mechanisms.

\section{Power divergence}

Finally, the effectiveness of a single branch could be demonstrated also in entropy terms. The logarithmic variance between the input and output terms will give the amount of divergence between these two.

The entropy of a single path $(w=1)$ for a unit power input $\left(P_{\text {in }}=1\right)$ is

$$
\begin{aligned}
e & =-\eta \log _{2} \eta, \quad \text { or } \\
& =-\eta \log _{2} \frac{P_{\text {out }}}{P_{\text {in }}}
\end{aligned}
$$

The term $\log _{2} \frac{P_{\text {out }}}{P_{\text {in }}}$ may be called the power divergence.

$$
e=-\eta\left(\log _{2} P_{\text {out }}-\log _{2} P_{\text {in }}\right)
$$

With the introduction of the entities $e_{\mathrm{o}}$ and $e_{\mathrm{i}}$, the output and the input power entropies respectively,

$$
\begin{aligned}
e_{\mathrm{o}} & =P_{0} \log _{2} P_{0} \quad \text { and } \\
e_{\mathrm{i}} & =P_{\text {in }} \log _{2} P_{\text {in }} \\
e & =-\left(\frac{e_{\mathrm{o}}}{P_{\text {in }}}-\eta \log _{2} P_{\text {in }}\right) \\
& =-\left(\frac{e_{\mathrm{o}}}{P_{\text {in }}}-\eta \frac{e_{\text {in }}}{P_{\text {in }}}\right) \\
e & =-P_{\text {in }}^{-1}\left(e_{\mathrm{o}}-\eta e_{\mathrm{i}}\right)
\end{aligned}
$$

But now consider the convention that the input power is taken unity. Then the above formulations may be reiterated as follows:

$$
\begin{aligned}
& \log _{2} \frac{P_{\text {out }}}{P_{\text {in }}}=\log _{2} P_{\text {out }} \\
& e=-\eta \log _{2} \eta=e=-\eta \log _{2} P_{\text {out }}
\end{aligned}
$$

hence,

$$
e=-e_{\mathrm{o}}
$$

This is to say that entropy across a single unit could be reduced by decreasing the output power entropy, i.e. by keeping the efficiency high.

\section{Conclusions}

The classical design concept have always tended to ignore the transmission junctions and labelled them by a simple constant. That concept has also regarded the power transfer phenomenon as a deterministic event in a narrow simplistic view. However, power transfer is a highly stochastic and conditional property of the systems that perform this transmittal. Changing working conditions, imperfect manufacturing techniques, varying loads all affect the constant that distinguishes the locality. This study has presented a new approach in the design of mechanical transmissions. The idea of transmission entropy is the novelty of this paper, and, to the knowledge of the author, this is the first time it is used in literature in this context. Also in this paper, entropy of the same system in two distinct formats was stated and compared. An optimization expression, 
i.e. cost function, was deduced for implementation for possible power flow route simulations. Nevertheless, the validity of the expressions, and the validity of conditions and assumptions must be verified on a worked example. Even though the optimization aspect of entropy is quite appealing, and was shortly formulated here, feasibility of such an application must also be proven for a wider acceptance.

\section{References}

[1] A. Cho, A fresh take on disorder or disorderly science, Science 297 (August) (2002) 1268-1269.

[2] I. Grabec, Absolute maximum entropy principle and self-organization of formal neurons, Open Systems and Information Dynamics 5 (1998) 279-288.

[3] V. Zhukov, V. Mizonov, P. Filitchev, S. Bernotat, The modeling of grinding processes by means of the principle of maximum entropy, Powder Technology 95 (1998) 248-253.

[4] M. Levene, G. Loizou, Computing the entropy of user navigation in the Web, University Report, University of London, 1999.

[5] K. Ziha, Event oriented system analysis, Probabilistic Engineering Mechanics 15 (2000) 261-275.

[6] C. Knessl, Integral representations and asymptotic expansions for Shannon and Renyi entropies, Applied Mathematics Letters 11 (2) (1998) 69-74.

[7] S. Ozdemir, J. Schueller, A new hybrid CVT design: CVPSTs, heavy vehicle systems, A Series of the International Journal of Vehicle Design 9 (4) (2002) 319-332. 